\title{
On interval pseudo-homogeneous uninorms
}

\author{
Lucelia Lima Costa $^{1}$ Benjamin Bedregal ${ }^{2}$ Humberto Bustince $^{3}$ Marcus Rocha $^{4}$ \\ ${ }^{1}$ Graduate program in Electrical Engineering and Computing, UFRN \\ ${ }^{2}$ Department of Informatics and Applied Mathematics, UFRN \\ ${ }^{3}$ Departamento de Automatica y Computacion, UPNA \\ ${ }^{4}$ Graduate program in Mathematics and statistics, UFPA
}

\begin{abstract}
In this paper, we introduce the concept of interval pseudo-homegeneous uninorms. We extend the concept of pseudo-homogeneity of specific functions for interval pseudo-homogeneous functions. It is studied two cases of interval pseudo-homogeneous uninorms, that is, interval pseudo-homogeneous tnorms and interval pseudo-homogeneous t-conorms. It is proved a form of interval pseudo-homogeneous t-norms, that is, $\mathbb{T}_{M}$ and we also prove that only interval t-conorm which is pseudo-homogeneous is $\mathbb{S}_{M}$ and that there are no interval pseudo-homogeneous proper uninorms.
\end{abstract}

Keywords: T-norm, t-conorm, uninorm, pseudohomogeneous

\section{Introduction}

Uninorms are a specific kind of aggregation operators that have proved to be useful in many fields like expert systems, aggregation, neural networks, and fuzzy system modeling. It is well known that a uninorm $\mathrm{U}$ can be a triangular norm or a triangular conorm whenever $U(1,0)=0$ or $U(1,0)=1$, respectively. They are interesting because of their structure as a specific combination of a t-norm and a t-conorm [11], [13].

T-norms and t-conorms have been introduced by Menger [18] and Schweizer and Sklar [22] in the context of the theory of probabilistic metric spaces and in this sense, have found applications in other areas such as the theory of fuzzy sets.

In Mathematics a homogeneous function is a function with conduct scalar multiplicative, i.e., if the arguments are multiplied by a factor, then the result is multiplied by a power of this factor. Generalized homogeneous t-norms (or t-conorms) should reflect the multiplicative constant $\lambda$ as well as the original value $T(x, y)$ or $S(x, y)$, and thus it should be expressed in the form $T(\lambda x, \lambda y)=F(\lambda, T(x, y))$ or $S(\lambda x, \lambda y)=F(\lambda, S(x, y))$.

Ebanks [8] generalized the concept of homogeneous t-norms, which is called quasi-homogeneous t-norms that are defined by a particular function $G(x, y)=\varphi^{-1}(f(x) \varphi(y))$, namely, $T(\lambda x, \lambda y)=$ $\varphi^{-1}(f(\lambda) \varphi(T(x, y)))$ for all $x, y, \lambda \in[0,1]$, where $f:[0,1] \rightarrow[0,1]$ is an arbitrary function and $\varphi$ is a strictly monotone and continuous function.
Considering function $G$ in the definition of quasihomogeneous t-norms, Xie [23] generalized it to more general functions and then introduced the concept of pseudo-homogeneous t-norms, t-conorms and proper uninorms.

Based on what was mentioned above, we naturally want to extend the concept of pseudohomogeneity of specific functions for interval pseudo-homogeneous functions, more precisely, interval pseudo-homogeneous uninorms. This is the motivation of the paper. It is showed two cases of interval pseudo-homogeneous uninorms, i. e., interval pseudo-homogeneous t-norms and interval pseudo-homogeneous t-conorms. Besides we prove that any interval proper uninorm is not interval pseudo-homogeneous.

\section{Preliminaries}

In this section, we recall the concepts of t-norms, tconorms, uninorms, pseudo-homogeneity and some results which will be used in the text.

Let $\mathbb{U}=\{[x, y] / 0 \leq x \leq y \leq 1\}$ be the set of closed subintervals of $[0,1]$. $\mathbb{U}$ is associated with two projections: $\Pi_{1}: \mathbb{U} \rightarrow[0,1]$ and $\Pi_{2}: \mathbb{U} \rightarrow[0,1]$ defined by

$$
\Pi_{1}([\underline{x}, \bar{x}])=\underline{x} \text { and } \Pi_{2}([\underline{x}, \bar{x}])=\bar{x}
$$

By convention, for any interval variable $X \in \mathbb{U}$, $\Pi_{1}(X)$ and $\Pi_{2}(X)$ will be denoted by $\underline{x}$ and $\bar{x}$, respectively.

Definition 2.1 An interval $X \in \mathbb{U}$ is strictly positive if and only if, $\underline{x}>0$. The set of strictly positive intervals in $\mathbb{U}$ will be denoted by $\mathbb{U}^{+}$

In [21], correctness was formalized through the notion of interval representation, where an interval function $F: \mathbb{U}^{n} \rightarrow \mathbb{U}$ represents a function $f:[0,1]^{n} \rightarrow[0,1]$ if for each $X \in \mathbb{U}^{n}, f(x) \in F(X)$ whenever $x \in X$ (the interval $X$ represents a $\mathrm{x}$ ).

On the other hand, if the functions $f, g:[0,1]^{n} \rightarrow$ $[0,1]$ are not asymptotic ${ }^{1}$ then the function $\widehat{f g}$ : $\mathbb{U}^{n} \rightarrow \mathbb{U}$ with $f \leq g$ defined by

\footnotetext{
${ }^{1}$ For us, a real function $f$ is asymptotic if for some interval $\left[a_{1}, b_{1}\right], \cdots,\left[a_{n}, b_{n}\right]$, the set $\left\{f\left(x_{1}, \cdots, x_{m}\right) / a_{j} \leq x_{j} \leq\right.$ $b_{j}$ for all $\left.j=1, \cdots, m\right\}$ either does have not supremum or does have not infimum.
} 


$$
\begin{aligned}
\widehat{f g}\left(X_{1}, \cdots, X_{n}\right)= & {\left[\operatorname { i n f } \left\{f\left(x_{i}, \cdots, x_{n}\right) /\right.\right.} \\
& \left.x_{i} \in X_{i} \text { for } i=1, \cdots, n\right\}, \\
& \sup \left\{g\left(x_{1}, \cdots, x_{n}\right) /\right. \\
& \left.\left.x_{i} \in X_{i} \text { for } i=1, \cdots, n\right\}\right]
\end{aligned}
$$

is well defined and it is an interval representation of every function $h: \mathbb{U}^{n} \rightarrow \mathbb{U}$ such that $f \leq h \leq g[21]$. When $\mathrm{f}$ and $\mathrm{g}$ are increasing we have $\widehat{f g}(X)=\left[f\left(\underline{x}_{1}, \cdots, \underline{x}_{n}\right), g\left(\bar{x}_{1}, \cdots, \bar{x}_{n}\right)\right]$. It is clear that, if $F$ is also an interval representation of $f: \mathbb{U}^{n} \rightarrow \mathbb{U}$, then for each $X \in \mathbb{U}^{n}, \widehat{f}(X) \subseteq F(X)$. When $f=g$ we will denote $\widehat{f g}$ by $\widehat{f}$. Clearly, $\widehat{f}$ returns a narrower interval than any other interval representation of $f$ and $\widehat{f}$ is therefore its best interval representation.

We define on $\mathbb{U}$ some partial orders:

Product order or Kulisch Miranker order: $X \leq$ $Y \Longleftrightarrow \underline{x} \leq \underline{y}$ and $\bar{x} \leq \bar{y}$;

Inclusion order: $X \subseteq Y \Longleftrightarrow y \leq \underline{x}$ and $\bar{x} \leq \bar{y}$;

Next, we define other operations that will be useful in this paper.

Definition 2.2 (Interval Product) Let $X$ and $Y$ be intervals, then the product of those intervals is defined by $X \cdot Y=[\underline{x} \underline{y}, \bar{x} \bar{y}]$, when $X \geq[0,0]$ and $Y \geq[0,0]$.

The interval product has the following algebraic properties: associativity, commutativity, the neutral element is the $\mathbf{1}=[1,1]$, subdistributivity with respect to the sum and $X \cdot[0,0]=[0,0]$.

Definition 2.3 (Interval Power) Let $X$ and $\mathbb{K}$ be strictly positive interval. The interval power of $X$ is given by $X^{\mathbb{K}}=\left\{x^{k} / x \in X\right.$ and $\left.k \in \mathbb{K}\right\}=$ $\left[\underline{x}^{\bar{k}}, \bar{x}^{\underline{k}}\right], 0 \leq \underline{k} \leq \bar{k} \leq 1$.

Observation 2.1 Observe that when $X, Y \in \mathbb{U}$, $X^{K_{1}} \cdot X^{K_{2}}=X^{K_{1}+K_{2}}$ and $(X Y)^{\mathbb{K}}=X^{\mathbb{K}} Y^{\mathbb{K}}$.

Proposition 2.1 [2, Theorem 4.2] Let $f, g$ : $[0,1]^{n} \rightarrow[0,1]$ such that $f \leq g . \widehat{f, g}$ is Moore continuous if and only if $f$ and $g$ are continuous.

Definition 2.4 $A$ t-norm is a function $T$ : $[0,1]^{2} \rightarrow[0,1]$ which satisfies the conditions of symmetry, associativity, monotonicity and has 1 as neutral element.

Example 2.1 Typical examples of t-norms are:

i) $T_{M}(x, y)=\min (x, y)$;

ii) $T_{P}(x, y)=x y$;

iii) $T_{W}(x, y)=\min (x, y)$ if $\max (x, y)=1$ and $T_{W}(x, y)=0$ otherwise.

Let $T_{1}$ and $T_{2}$ be t-norms, we have $T_{1} \leq T_{2}$ if for every $x, y \in[0,1], T_{1}(x, y) \leq T_{2}(x, y)$.
Definition 2.5 [5] A function $\mathbb{T}: \mathbb{U}^{2} \rightarrow \mathbb{U}$ is an interval $t$-norm if $\mathbb{T}$ is symmetric, associative, monotonic with respect to the order of Kulisch -Miranker, and [1,1] is the neutral element.

Definition 2.6 [6] An interval t-norm $\mathbb{T}$ is $t$ representable if there exist $t$-norms $T_{1}$ and $T_{2}$ such that $T_{1} \leq T_{2}$ and $\mathbb{T}=\widehat{T_{1} T_{2}}$.

Definition 2.7 [5] An interval t-norm $\mathbb{T}$ is inclusion monotonic if $\forall X, Y, Z \in \mathbb{U}, \mathbb{T}(X, Y) \subseteq \mathbb{T}(X, Z)$ when $Y \subseteq Z$.

Theorem 2.1 ([9], Corollary 33) An interval $t$ norm $\mathbb{T}: \mathbb{U}^{2} \rightarrow \mathbb{U}$ is t-representable if and only if it is inclusion monotonic.

Let $\mathbb{T}_{1}$ and $\mathbb{T}_{2}$ be interval t-norms. We have $\mathbb{T}_{1} \leq$ $\mathbb{T}_{2}$ if for every $X, Y \in \mathbb{U}, \mathbb{T}_{1}(X, Y) \leq \mathbb{T}_{2}(X, Y)$.

Proposition 2.2 Let $T_{1}$ and $T_{2}$ be t-norms. $T_{1} \leq$ $T_{2}$ if and only if $\widehat{T_{1}} \leq \widehat{T_{2}}$.

Proof: $(\Rightarrow)$ See [3], Proposition 5.1.

$(\Leftarrow)$ Let $x, y \in[0,1]$. Then

$$
\widehat{T_{1}}([x, x],[y, y]) \leq \widehat{T_{2}}([x, x],[y, y])
$$

or in other words,

$$
\left[T_{1}(x, y), T_{1}(x, y)\right] \leq\left[T_{2}(x, y), T_{2}(x, y)\right]
$$

Thus,

$$
T_{1}(x, y) \leq T_{2}(x, y)
$$

Similarly to the case of t-norms, many classes of interval t-norms can be defined [3]. We examined only some of them, for example, interval tnorms that have zero divisors, interval Archimedean t-norms and interval idempotent t-norms.

An interval t-norm $\mathbb{T}$ has zero divisors if there is at least one pair of elements $X \neq[0,0]$ and $Y \neq[0,0]$, such that $\mathbb{T}(X, Y)=[0,0]$. For example, $\widehat{T_{W}}([0.4,0.9],[0.6,0.7])=[0,0]$. If an interval t-norm has no zero divisor then $\mathbb{T}(X, Y)=\mathbf{0}$ if and only if $X=[0,0]$ or $Y=[0,0]$.

Let $\mathbb{T}$ be an interval t-norm. $\mathbb{T}$ is Archimedean if for each $X, Y \in \mathbb{U}-\{[0,0],[1,1]\}$, there exists a positive integer $n$ such that $X^{(n)}<Y$ where $X^{(1)}=$ $X$ and $X^{(k+1)}=\mathbb{T}\left(X, X^{(k)}\right)$.

An interval t-norm is idempotent if $\mathbb{T}(X, X)=X$ for all $X \in \mathbb{U}$, for example $\mathbb{T}_{M}$, where $\mathbb{T}_{M}(X, Y)=$ $[\min (\underline{x}, \underline{y}), \min (\bar{x}, \bar{y})]$.

Proposition 2.3 [7] The only interval t-norm which is idempotent is $\mathbb{T}_{M}$.

Definition 2.8 A triangular conorm is a function $S:[0,1]^{2} \rightarrow[0,1]$ that is symmetric, associative, monotonic and has 0 as neutral element. 
A t-norm $T$ and a t-conorm $S$ are dual with respect to $N(x)=1-x$ when $T(x, y)=1-S(1-x, 1-y)$ for all $x, y \in[0,1]$.

Example 2.2 An example of a basic t-conorm is: $S_{M}(x, y)=\max (x, y)$

Definition 2.9 [5] A function $\mathbb{S}: \mathbb{U}^{2} \rightarrow \mathbb{U}$ is an interval t-conorm if $\mathbb{S}$ is symmetric, associative, monotonic and $[0,0]$ is the neutral element.

Definition 2.10 An interval t-conorm $\mathbb{S}$ is $s$ representable if there exist $t$-conorms $S_{1}$ and $S_{2}$ such that $S_{1} \leq S_{2}$ and $\mathbb{S}=\widehat{S_{1} S_{2}}$.

In [23] Xie at al. defined pseudo-homogeneous t-norms and t-conorms and constructed the tuple $(T, F)$ which satisfies the pseudo-homogeneous equation. Next, some of these results are showed.

Definition 2.11 [23] $A$ t-norm $T$ is said to be pseudo-homogeneous if it satisfies $T(\lambda x, \lambda y)=$ $F(\lambda, T(x, y))$ for all $x, y, \lambda \in[0,1]$, where $F$ : $[0,1]^{2} \rightarrow[0,1]$ is a continuous and increasing function with $F(x, 1)=0 \Leftrightarrow x=0$.

Lemma 2.2 Let $T$ be a pseudo-homogeneous $t$ norm. Then $T$ is positive.

\section{Proof}

Suppose that there exist $x, y \neq 0$ such that $T(x, y)=0$. Let $z=\min (x, y)$, then $z \neq 0$ and because $T$ is increasing, $T(z, z)=0$. Thus, $T(z, z)=F(z, T(1,1))=F(z, 1) \neq 0$ which is a contradiction.

Lemma 2.3 [23] If $T$ is a pseudo-homogeneous $t$ norm, then it must be continuous.

Lemma 2.4 [23] Let $T$ be a t-norm. Then $T(x, x y)=T(y, x y)$ for any $x, y \in[0,1]$ if and only if $T=T_{M}$.

Lemma 2.5 [23] Let $T$ be a pseudo-homogeneous t-norm and $F$ be the same as in Definition 2.11. Then $F$ is commutative if and only if $T(x, x)=x$ for any $x \in(0,1)$, i.e., $T=T_{M}$.

Definition 2.12 $A$ t-conorm $S$ is called pseudo-homogeneous if it satisfies $S(\lambda x, \lambda y)=$ $F(\lambda, S(x, y))$ for all $x, y, \lambda \in[0,1]$, where $F:[0,1]^{2} \rightarrow[0,1]$ is an increasing function.

Theorem 2.6 [23] $A$ t-conorm $S$ is pseudohomogeneous if and only if $S=S_{M}$ and $F(x, y)=$ $x y$.

Definition 2.13 A function $U:[0,1]^{2} \rightarrow[0,1]$ is called a uninorm if it is commutative, associative and increasing and has a neutral element $e \in[0,1]$.
There are two cases: if $e=1$, it leads back to t-norms. If $e=0$, it leads back to t-conorms. Any uninorm with neutral element in $(0,1)$ is called proper uninorm [11].

Definition 2.14 [23] A proper uninorm $U$ is called pseudo-homogeneous if it satisfies $U(\lambda x, \lambda y)=$ $F(\lambda, U(x, y))$ for all $x, y, \lambda \in[0,1]$, where $F$ : $[0,1]^{2} \rightarrow[0,1]$ is an increasing function.

Proposition 2.4 [23] Let $U$ be a proper uninorm. Then $U$ is never pseudo-homogeneous.

\section{Interval pseudo-homogeneous uninorms}

As previously stated any uninorm with neutral element $e \in(0,1)$ is called proper [11]. In this section, we introduce the concept of interval pseudohomogeneous uninorms and we show there are no interval pseudo-homogeneous proper uninorms.

Definition 3.1 [20] A function $\mathcal{U}: \mathbb{U}^{2} \rightarrow \mathbb{U}$ is called an interval uninorm if it is commutative, associative, increasing and has a neutral element $e \in \mathbb{U}$. When the neutral element $e$ is neither $[0,0]$ nor $[1,1]$ the interval uninorm $\mathcal{U}$ is called of proper.

Definition 3.2 A interval uninorm $\mathcal{U}$ is called interval pseudo-homogeneous if there exist $\mathbb{F}: \mathbb{U}^{2} \rightarrow \mathbb{U}$ such that

$$
\mathcal{U}(\lambda X, \lambda Y)=\mathbb{F}(\lambda, \mathcal{U}(X, Y)),
$$

for all $X, Y, \lambda \in \mathbb{U}$.

Proposition 3.1 Let $\mathcal{U}$ be an interval uninorm with neutral element $e . \quad$ If $\mathcal{U}$ is interval pseudohomogeneous with respect to a function $\mathbb{F}: \mathbb{U}^{2} \rightarrow \mathbb{U}$, then $\mathbb{F}$ satisfies the following properties:

1. $\mathbb{F}([0,0], X)=\mathbb{F}(X,[0,0])=[0,0]$;

2. $\mathbb{F}$ is increasing;

3. $\mathbb{F}(e, Y) \leq e Y$;

4. $\mathbb{F}(X, e) \leq e X$.

\section{Proof}

1. $\mathbb{F}([0,0], X)=\mathbb{F}([0,0], \mathcal{U}(X, e))=$ $\mathcal{U}([0,0] X,[0,0] e)=\mathcal{U}([0,0],[0,0])=[0,0]$ and $\mathbb{F}(X,[0,0])=\mathbb{F}(X, \mathcal{U}([0,0],[0,0]))=$ $\mathcal{U}(X[0,0], X[0,0])=\mathcal{U}([0,0],[0,0])=[0,0]$.

2. If $Y \leq Z$ then $\mathbb{F}(X, Y)=\mathbb{F}(X, \mathcal{U}(Y, e))=\mathcal{U}(X Y, X e) \leq$ $\mathcal{U}(X Z, X e)=\mathbb{F}(X, \mathcal{U}(Z, e))=\mathbb{F}(X, Z)$

and $\mathbb{F}(Y, X)=\mathbb{F}(Y, \mathcal{U}(X, e))=\mathcal{U}(Y X, Y e) \leq$ $\mathcal{U}(Z X, Z e)=\mathbb{F}(Z, \mathcal{U}(X, e))=\mathbb{F}(Z, X)$.

3. $\mathbb{F}(e, Y)=\mathbb{F}(e, \mathcal{U}(Y, e))=\mathcal{U}\left(e Y, e^{2}\right) \leq$ $\mathcal{U}(e Y, e)=e Y$

4. $\mathbb{F}(X, e)=\mathbb{F}(X, \mathcal{U}(e, e))=\mathcal{U}(X e, X e) \leq$ $\mathcal{U}(X e, e)=X e$. 
Theorem 3.1 Let $\mathbb{T}$ be an interval t-norm. If $\mathbb{T}$ is interval pseudo-homogeneous with respect a function $\mathbb{F}: \mathbb{U}^{2} \rightarrow \mathbb{U}$ then $\mathbb{F}$ is an interval conjunctive aggregation function.

Proof From Proposition 3.1, $\mathbb{F}$ is increasing and $\mathbb{F}([0,0],[0,0])=[0,0]$. Since, $\mathbb{F}([1,1],[1,1])=$ $\mathbb{F}([1,1], \mathbb{T}([1,1],[1,1]))=\mathbb{T}([1,1],[1,1])=[1,1]$. Therefore, $\mathbb{F}$ is an aggregation function. In addition, because $\mathbb{F}$ is increasing and from Proposition 3.1, $\mathbb{F}(X, Y) \leq \mathbb{F}([1,1], Y) \leq[1,1] Y=Y$ and $\mathbb{F}(X, Y) \leq \mathbb{F}(X,[1,1]) \leq X[1,1]=X$. So, $\mathbb{F}(X, Y) \leq \inf (X, Y)$.

Proposition 3.2 Let $\mathcal{U}$ be an interval proper uninorm. Then $\mathcal{U}$ is never interval pseudohomogeneous.

\section{Proof}

Suppose that $\mathcal{U}$ is an interval pseudo-homogeneous proper uninorm with neutral element $e \in \mathbb{U}-$ $\{[0,0],[1,1]\}$. According to (1), we have

$$
\begin{aligned}
{[\underline{e}, \bar{e}]^{2} } & =\mathcal{U}([\underline{e}, \bar{e}][\underline{e}, \bar{e}],[\underline{e}, \bar{e}][1,1]) \\
& =\mathbb{F}([\underline{e}, \bar{e}], \mathcal{U}([\underline{e}, \bar{e}],[1,1])) \\
& =\mathbb{F}([\underline{e}, \bar{e}],[1,1]) .
\end{aligned}
$$

Therefore, $\mathbb{F}([\underline{e}, \bar{e}],[1,1])=[\underline{e}, \bar{e}]^{2}$.

$$
\begin{aligned}
{[\underline{e}, \bar{e}][1,1] } & =\mathcal{U}([\underline{e}, \bar{e}][1,1],[\underline{e}, \bar{e}][1,1]) \\
& =\mathbb{F}([\underline{e}, \bar{e}], \mathcal{U}([1,1],[1,1])) \\
& =\mathbb{F}([\underline{e}, \bar{e}],[1,1])=[\underline{e}, \bar{e}]^{2}
\end{aligned}
$$

which is a contradiction. Thus, there is no interval proper uninorm which is interval pseudohomogeneous.

\subsection{Two cases of interval pseudo-homogeneous uninorms}

Uninorms are a generalization of both t-norms and t-conorms [24]. Here we show that when $e=[1,1]$ we have an interval pseudo-homogeneous t-norm and when $e=[0,0]$ we have an interval pseudohomogeneous t-conorm. In this sense, there are only two cases of interval pseudo-homogeneous uninorms.

Definition 3.3 [17] A interval t-norm $\mathbb{T}$ is said to be interval pseudo-homogeneous if it satisfies

$\mathbb{T}(\lambda X, \lambda Y)=\mathbb{F}(\lambda, \mathbb{T}(X, Y))$ for all $X, Y, \lambda \in \mathbb{U}$,

where $\mathbb{F}: \mathbb{U}^{2} \rightarrow \mathbb{U}$ is a Moore continuous and increasing function with $\mathbb{F}(X,[1,1])=[0,0] \Leftrightarrow X=$ $[0,0]$.
Observation 3.1 The above definition is a specific case of Definition 3.2, since all interval $t$-norm are interval uninorms and any function $\mathbb{F}$ with the above condition, also satisfies Definition 3.2.

Lemma 3.2 Let $\mathbb{T}$ be an interval pseudohomogeneous t-norm. Then $\mathbb{T}$ has no zero divisors.

\section{Proof}

Suppose that there exist $X, Y \neq[0,0]$ such that $\mathbb{T}(X, Y)=[0,0]$. Let $Z=\inf (X, Y)$. Then, $Z \neq[0,0]$ and, because $\mathbb{T}$ is increasing, $\mathbb{T}(Z, Z)=[0,0]$. On the other hand, $\mathbb{T}(Z, Z)=$ $\mathbb{F}(Z, \mathbb{T}([1,1],[1,1]))=\mathbb{F}(Z,[1,1]) \neq[0,0]$ which is a contradiction.

Lemma 3.3 [17] Let $\mathbb{T}$ be an interval pseudohomogeneous t-norm with respect to a function $\mathbb{F}$. If $\mathbb{T}$ is t-representable then there exist continuous and increasing functions $F_{1}, F_{2}:[0,1]^{2} \rightarrow[0,1]$ satisfying the condition $F_{i}(x, 1)=0 \Leftrightarrow x=0$ and such that $\mathbb{F}=\widehat{F_{1} F_{2}}$.

Theorem 3.4 [17] Let $\mathbb{T}$ be a t-representable interval t-norm. $\mathbb{T}$ is interval pseudo-homogeneous if and only if its represents are pseudo homogeneous.

Lemma 3.5 Let $\mathbb{T}$ be a t-norm. Then $\mathbb{T}(X, X Y)=$ $\mathbb{T}(Y, X Y)$ for any $X, Y \in \mathbb{U} \Leftrightarrow \mathbb{T}=\mathbb{T}_{M}$.

\section{Proof}

$(\Rightarrow)$ Suppose that $\mathbb{T}(X, X Y)=\mathbb{T}(X Y, Y)$. By fixing $X=[1,1]$, we get that $\mathbb{T}(1, Y)=\mathbb{T}(Y, Y)$ for any $Y \in \mathbb{U}$. Thus $\mathbb{T}(Y, Y)=Y$.

$(\Leftarrow)$ On the other hand, if $\mathbb{T}=\mathbb{T}_{M}$, then $\mathbb{T}(X, X Y)=X Y=\mathbb{T}(X Y, Y)$.

Proposition 3.3 Let $\mathbb{T}$ be an interval pseudohomogeneous t-norm and $\mathbb{F}$ be the same as in Definition 3.3. Then $\mathbb{F}$ is commutative if and only if $\mathbb{T}(X, X)=X$ for any $X \in \mathbb{U}$. i.e., $\mathbb{T}=\mathbb{T}_{M}$.

Proof

By Eq. (2), we have $\mathbb{T}(X, X Y)=\mathbb{F}(X, Y)$ for any $X, Y \in \mathbb{U}$. Similary, $\mathbb{T}(X Y, Y)=\mathbb{F}(Y, X)$ for any $X, Y \in \mathbb{U}$. Therefore,

$$
\begin{aligned}
\mathbb{F} \text { is commutative } & \Leftrightarrow \mathbb{F}(X, Y)=\mathbb{F}(Y, X) \forall X, Y \in \mathbb{U} \\
& \Leftrightarrow \mathbb{T}(X, X Y)=\mathbb{T}(X Y, Y) \forall X, Y \in \mathbb{U} \\
& \Leftrightarrow \mathbb{T}=\mathbb{T}_{M} \text { by Lemma } 3.5 \\
& \Leftrightarrow \mathbb{T}(X, X)=X \forall X \in \mathbb{U} \text { by Prop. } 2.3
\end{aligned}
$$

Observe that there are interval pseudohomogeneous interval t-norms in the sense of Def.3.2 which are not interval pseudo-homogeneous in the sense of Def. 3.3, e.g. the interval t-norm 
$\mathbb{T}(X, Y)=\left\{\begin{array}{cc}{[0,0]} & \text { if } \sup (X, Y)<[1,1] \\ \inf (X, Y) & \text { otherwise }\end{array}\right.$

Alsina at al. [1] proved that if $S$ is a homogeneous t-conorm, then k=1 and $S=S_{M}$. Here, we will extend the concept of pseudo-homogeneous t-conorm and will show that the only interval t-conorm which is pseudo-homogeneous is $\mathbb{S}_{M}$.

Definition 3.4 $A$ t-conorm $\mathbb{S}$ is called interval pseudo-homogeneous if it satisfies $\mathbb{S}(\lambda X, \lambda Y)=$ $\mathbb{F}(\lambda, \mathbb{S}(X, Y))$ for all $X, Y, \lambda \in \mathbb{U}$, where $\mathbb{F}: \mathbb{U}^{2} \rightarrow \mathbb{U}$ is an increasing function.

Theorem 3.6 A interval t-conorm $\mathbb{S}$ is pseudohomogeneous if and only if $\mathbb{S}=\mathbb{S}_{M}$ and $\mathbb{F}(X, Y)=$ $X Y$.

\section{Proof}

$(\Rightarrow)$ Suppose that for some increasing function $\mathbb{F}$ : $\mathbb{U}^{2} \rightarrow \mathbb{U}, \mathbb{S}$ satisfies

$$
\mathbb{S}(\lambda X, \lambda Y)=\mathbb{F}(\lambda, \mathbb{S}(X, Y))
$$

for all $X, Y, \lambda \in \mathbb{U}$, then for any $X \in \mathbb{U}$

$$
\begin{aligned}
\mathbb{S}(X, X) & =\mathbb{S}(X \cdot[1,1], X \cdot[1,1]) \\
& =\mathbb{F}(X, \mathbb{S}([1,1],[1,1])) \\
& =\mathbb{F}(X,[1,1])
\end{aligned}
$$

and

$$
\begin{aligned}
X=\mathbb{S}([0,0], X) & =\mathbb{S}(X \cdot[0,0], X \cdot[1,1]) \\
& =\mathbb{F}(X, \mathbb{S}([0,0],[1,1])) \\
& =\mathbb{F}(X,[1,1]),
\end{aligned}
$$

Thus, $\mathbb{S}(X, X)=X$ for any $X \in \mathbb{U}$ and $\mathbb{S}=\mathbb{S}_{M}$. Moroever, since

$$
\begin{aligned}
X Y & =\mathbb{S}(X Y, X Y) \\
& =\mathbb{F}(X, \mathbb{S}(Y, Y)) \\
& =\mathbb{F}(X, Y)
\end{aligned}
$$

Thus,

$$
\mathbb{F}(X, Y)=X Y
$$

$(\Leftarrow)$ Let $\mathbb{S}=\mathbb{S}_{M}$ and $\mathbb{F}(X, Y)=X Y$, then $\mathbb{S}(\lambda X, \lambda Y)=\sup (\lambda X, \lambda Y)=\lambda \sup (X, Y)$ and

$$
\begin{aligned}
\mathbb{F}(\lambda, \mathbb{S}(X, Y)) & =\mathbb{F}(\lambda, \sup (X, Y)) \\
& =\lambda \sup (X, Y)
\end{aligned}
$$

Thus,

$$
\mathbb{S}(\lambda X, \lambda Y)=\mathbb{F}(\lambda, \mathbb{S}(X, Y)),
$$

for all $X, Y, \lambda \in \mathbb{U}$.

\section{Final remarks}

In this paper, we consider the study of interval pseudo-homegeneous functions, but specifically the pseudo-homogeneous uninorms. It is studied two cases of interval pseudo-homogeneous uninorms, i.e., interval pseudo-homogeneous t-norms and interval pseudo-homogeneous t-conorms. It is proved a form of interval pseudo-homogeneous t-norms, i.e., $\mathbb{T}_{M}$ and we also proved that only interval t-conorm which is pseudo-homogeneous is $\mathbb{S}_{M}$ and that there are no interval pseudo-homogeneous proper uninorms.

Since in [23] it has been proved that exist two more forms of pseudo-homogeneous t-norms, but in work we proved only one for interval case. Then, in the future work, we will prove these two forms for interval case.

\section{Acknowledgments}

This work is supported by the Brazilian funding agencies CNPq (Ed. PQ and PVE, under the process numbers 307681/2012-2 and 406503/2013-3, respectively and SWE 202606/2014-7) and also by the project TIN2013-40765-P of the Spanish Ministry of Science.

\section{References}

[1] Alsina, C.,Frank, M. J. \& Schweizer, B.: Associative functions. Triangular norms and copulas. World Scientific Publishing Co., Singapore, 2006.

[2] Bedregal, B. R. C.: On interval fuzzy negations. Fuzzy Sets and Systems, 161(17):22902313, 2010.

[3] Bedregal, B. R. C. \& Takahashi, A.: Interval tnorms as interval representations of t-norms, in: Proceedings of the IEEE International Conference on Fuzzy Systems, Reno, IEEE, Los Alamitos, pp. 909-914, 2005.

[4] Bedregal, B. R. C. \& Takahashi, A.: Interval valued of $\mathrm{t}$-conorms, fuzzy negations and fuzzy aplications, in:Proceedings of the IEEE International Conference on Fuzzy systems, Vancouver, IEEE, Los Alamitos, pp 1981-1987, 2006.

[5] Bedregal, B. R. C.\& Takahashi, A.: T-norms, t-conorms, complements and interval implications. Tema Tend. Mat. Apl. Comput., 7 (1) 139-148, 2006.

[6] Bedregal, B. R. C.\& Takahashi, A.: The best interval representations of t-norms and automorphisms. Fuzzy Sets and Systems. 157: 32203230, 2006.

[7] Bustince, H., Barrenechea, E. \& Pagola M.: Generation of interval-valued fuzzy and Atanassov's intuitionistic fuzzy connectives from fuzzy connectives and from $K_{\alpha}$ operators: Laws for conjunctions and disjunctions, ampli- 
tude, International journal of intelligent systems, (23), 680-714, 2008.

[8] Ebanks, B. R.: Quasi-homogeneous associative functions. Internal. J. Math. Sci. 21, 351-358, 1998.

[9] Dimuro, G. P, Bedregal, B. C., Santiago, R. H. N. \& Reiser, R. H. S.: Interval additive generators of interval t-norms and interval t-conorms. Information Sciences 181: 3898-3916, 2011.

[10] Dubois, D.\& Prade, H.: Random sets and fuzzy interval analysis. Fuzzy Sets and Systems, 42:87-101, 1991.

[11] Fodor, J.: De Baets, Uninorms basics, in: P.P. Wang, E.E. Kerre (Eds.), Fuzzy Logic: A Spectrum of Theoretical and Practical Issues, in: Studies in Fuzziness and Soft Computing, vol. 215, Springer, Berlin, 49-64, 2007.

[12] Gomez, D. \& Montero, J.: A discussion on aggregation operators. Kybernetika, 40: 107-120, 2004.

[13] Klement, E. P., Mesiar, R. \& Pap, E.: Triangular norms. Kluwer Academic Publishers, Dordrecht, 2000.

[14] Klement, E. P., Mesiar, R. \& Pap, E.: Triangular norms. Position paper I: Basic analytical and algebraic properties. Fuzzy Sets and Systems, 143(1): 5-26, 2004.

[15] Klement, E. P., Mesiar, R. \& Pap, E.: Triangular norms. Position paper II: General construcions and parameterized families. Fuzzy Sets and Systems, 145(3): 411-438, 2004.

[16] Klement, E. P., Mesiar, R. \& Pap, E.: Triangular norms. Position paper III: Continuous t-norms. Fuzzy Sets and Systems, 145(3): 439454, 2004.

[17] Lima, L., Bedregal, B., Bustince, H., Barrenechea, E. \& Rocha, M.: An extension of homogeneous and pseudo-homogeneous functions with applications. submitted to the jornal, 2015.

[18] Menger, K.: Statistical metrics. Proc. Nat. Acad., pp. 535-537, 1942.

[19] Moore, R., Kearfott, R. B. \& Cloud, M. J.: Introduction to interval analysis. Studies in Applied Mathematics. SIAM, Philadelphia, 2009.

[20] Santana, F. L., Santiago, R. H. N. \& Santana, F. T.: On monotonic inclusion interval uninorms. Conference: The 11th International, FLINS, 2014.

[21] Santiago, R., Bedregal, B.\& Acioly, B.: Formal aspects of correctness and optimality of interval computations. Formal Aspects of Computing, 2005.

[22] Schweizer, B. \& Sklar, A.: Associative functions and abstract semigroups. Publ. Math. Debrecen, 10, 69-81, 1963.

[23] Xie, A., Su, Y. \& Liu, H.: On pseudohomogeneous triangular norms, triangular conorms and proper uninorms.Fuzzy Sets and Systems, 2014.

[24] Yager, R. \& Rybalov, A.: Uninorm aggregation operators, Fuzzy Sets and Systems. 80, 111-120, 1996.

[25] Zadeh, L. A.: Fuzzy sets. Information and Control. 8: 338-353, 1965. 